\title{
Analyse de la gouvernance systémique des universités au Québec et comparaisons avec quatre autres provinces canadiennes
}

Olivier Bégin-Caouette

Université de Montréal

Claude Trottier

Université Laval

Julia Eastman

University of Victoria

Glen A. Jones

University of Toronto

Christian Noumi

University of Toronto

Sharon X. Li

University of Toronto

\section{Résumé}

Les sociétés du savoir ont de nombreuses exigences à l'égard des universités ; or il est suggéré que ces dernières répondraient plus adéquatement à ces exigences lorsqu'elles sont autonomes (Altbach et Balán, 2007). Cet article vise à mettre en lumière diverses dimensions de l'encadrement du système universitaire québécois par l'État relativement à l'autonomie des établissements et à comparer la situation québécoise avec celle ayant cours en Alberta, en Colombie-Britannique, en Ontario et en Nouvelle-Écosse. Une analyse thématique réalisée sur 77 documents officiels (dont 29 au Québec) et sur les transcriptions de 93 entrevues (dont 30 au Québec) a 
permis d'identifier trois grandes dimensions de la gouvernance systémique, soit le cadre législatif et réglementaire, le statut comptable et les règles budgétaires, ainsi que les interventions du gouvernement dans la gestion interne des universités. L'analyse révèle que l'autonomie dont jouissent les universités varie d'une province à l'autre : les universités néo-écossaises et ontariennes bénéficieraient de plus d'autonomie que leurs consœurs albertaines et britanno-colombiennes. Le Québec se situerait globalement au centre puisqu'il comprend deux secteurs universitaires dont les dimensions suggèrent des niveaux d'autonomie différents.

\begin{abstract}
Knowledge societies have expectations of universities, and it has been suggested that autonomy contributes to universities' capacity to respond adequately (Altbach \& Balán, 2007). This article aims to highlight the dimensions of state oversight and its relation to institutions' autonomy in Quebec, and to compare the situation with that in Alberta, British Columbia, Ontario and Nova Scotia. A thematic analysis of 77 official documents (including 29 in Quebec) and 93 interview transcripts (including 30 in Quebec) led to the identification of three broad dimensions of systemic governance: the legislative and regulatory framework, universities' accounting status and governments' budgetary rules, as well as government interventions in universities' internal management. The analysis reveals that university autonomy varies by province, with universities in Nova Scotia and Ontario being possibly more autonomous than universities in Alberta and British Columbia; Quebec would be in-between, partly because it includes two university sectors whose dimensions suggest different levels of autonomy.
\end{abstract}

En tant qu'acteurs-clés des sociétés du savoir, les universités en sont venues à occuper l'avant-plan de nombreuses politiques publiques exigeant qu'elles soient accessibles, imputables et pertinentes par rapport aux attentes de la société (Metcalfe, Fisher, Gingras, Jones, Rubenson et Snee, 2011). Pour Estermann, Nokkala et Steinel (2011), les universités répondent plus adéquatement à ces demandes lorsqu'elles sont autonomes. Si l'autonomie est bénéfique pour l'ensemble des universités, Altbach et Balán (2007) affirment qu'elle l'est d'autant plus pour les universités de recherche dont le travail est plus spécialisé.

L'objectif de l'article est d'examiner la gouvernance systémique des universités au Québec et dela comparer à celle d'autres provinces canadiennes. Le concept de gouvernance systémique - qui sera défini ci-après - permet d'explorer comment, dans des contextes provinciaux qui ont connu une évolution historique différente, les interactions entre l'État et les universités influencent l'autonomie de ces dernières. Bien que nous reconnaissions l'influence d'une diversité d'acteurs dans la gouvernance universitaire, cet article porte particulièrement attention aux relations entre les universités et les gouvernements provinciaux puisque, comme le souligne Thorens (2006), si l'autonomie institutionnelle et la liberté universitaire dépendent du bon vouloir de la société, elles dépendent surtout du dépositaire du pouvoir politique, l'État. 


\section{Contexte}

Au Canada, les universités sont des corporations sans but lucratif établies par des chartes et des lois provinciales. À la suite de la Seconde Guerre mondiale, les gouvernements ont accru leurs investissements dans les universités et, n'ayant pas les capacités organisationnelles pour coordonner ce secteur en expansion, ont mis sur pied des commissions d'enquête (comme la Commission royale d'enquête sur l'enseignement dans la province de Québec ou le Rapport Deutsch au Nouveau-Brunswick), des organismes intermédiaires (comme le Conseil ontarien de la qualité de l'enseignement supérieur), des organismes-conseils (comme la Commission de l'enseignement supérieur des Provinces maritimes) et, à partir des années 1980, des formules de financement en partie basées sur la performance (Lang, 2016).

Malgré l'instauration de mécanismes de reddition de comptes, les comparaisons internationales menées par Richardson et Fielden (1997), de même que par Anderson et Johnson (1998), suggèrent que le niveau d'intervention des gouvernements au Canada était parmi les plus bas du monde. Plus récemment, Maheu et Lacroix (2015) estimaient aussi que les universités canadiennes avaient plus d'autonomie que leurs homologues d'autres pays (notamment de France). Comparer le Canada à d'autres pays demeure toutefois délicat puisque l'éducation est de juridiction provinciale. Même si le gouvernement fédéral intervient dans le financement de la recherche, la formation professionnelle, l'aide financière aux étudiants et l'éducation dans les communautés autochtones, il existe de fait dix systèmes d'enseignement supérieur au Canada, chacun structuré par des systèmes de gouvernance particuliers (Fisher et Rubenson, 2014; Shanahan etJones, 2007). Le système d'enseignement supérieur québécois est probablement celui qui se distingue le plus des autres provinces, notamment par la prédominance du français, son héritage catholique avant que les établissements d'enseignement supérieur ne soient déconfessionnalisés, le réseau des Collèges d'enseignement général et professionnel (CÉGEP), la coexistence du réseau de l'Université du Québec et de huit universités à charte, le bas niveau des droits de scolarité et le militantisme étudiant.

Au Québec, les premières universités francophones étaient catholiques et dirigées par des hommes d'Église. Dans la foulée de la Révolution tranquille, la Commission Parent proposa la création d'un ministère de l'Éducation du Québec (MEQ) incluant une direction de l'enseignement supérieur. Pour des raisons d'efficience et d'accessibilité, elle avait proposé une coordination accrue de l'enseignement supérieur, mais pour tenir compte des appréhensions des universités, le gouvernement créa des organismes consultatifs, tels que le Conseil supérieur de l'éducation en 1964 et le Conseil des universités en 1968, et laissa aux universités les responsabilités de se coordonner et de s'évaluer.

De toutes les transformations qui ont marqué le système d'enseignement supérieur québécois, c'est celle ayant trait à l'imputabilité qui a eu l'impact le plus direct sur la gouvernance du système et des établissements (Trottier et al., 2014). Le terme est apparu pour la première fois dans le Rapport de la Commission d'étude sur les universités (Gouvernement du Québec, 1979a), puis dans le Rapport du Comité d'étude sur l'université et la société québécoise (Gouvernement du Québec, 1979b) qui posa clairement l'enjeu $\mathrm{du}$ « conflit constant entre l'autonomie nécessaire aux universités pour accomplir leurs fonctions et les exigences de leurs responsabilités sociales à titre d'institutions publiques » (p. 63). 
La dissémination d'une idéologie qui prônait la réduction de la taille de l'État au début des années 1990 et au cours des années 2000 a aussi transformé la gouvernance des universités, notamment par l'abolition du Conseil des universités en 1993 et l'adoption de la Loi sur l'administration publique en 2000 qui, par ricochet, a eu un impact notable sur la régulation de l'enseignement supérieur. Premièrement, le Conseil des universités exerçait une influence considérable sur l'élaboration des politiques relatives à la coordination, la planification et le financement du système universitaire de même que sur l'évaluation de la qualité des programmes. Son abolition permit au MEQ de récupérer une partie des prérogatives du Conseil et à la Conférence des recteurs et principaux des universités du Québec (CREPUQ) de se doter d'une Commission de vérification de l'évaluation des programmes existants et d'une Commission d'évaluation des projets de programmes.

Deuxièmement, le gouvernement modifia en 1995 la Loi sur les établissements de niveau universitaire pour qu'ils rendent compte de leur administration en joignant à leurs états financiers annuels un rapport sur le salaire de leurs hauts dirigeants et leur performance, notamment en ce qui concerne le taux de réussite des étudiants, les moyens mis en œuvre pour les supporter de même que sur leurs perspectives de développement. Ces rapports sont examinés annuellement par la Commission de la culture et de l'éducation de l'Assemblée nationale. Les dirigeants des universités doivent, de surcroît, se présenter devant cette commission au moins une fois tous les trois ans (Trottier et al., 2014). Troisièmement, inspirée par les principes sous-jacents à la Loi sur l'administration publique, la Politique québécoise de financement des universités (Gouvernement du Québec, 2000) prévoyait que le financement additionnel prévu dans les budgets 20012004 serait conditionnel à la conclusion de contrats de performance de trois ans. Cette mesure fut abolie par le Parti libéral en 2005 qui ne l'octroya qu'en retour de l'engagement des universités d'équilibrer leur budget à court terme. Le contrôle gouvernemental s'est à nouveau renforcé avec les modifications à la Loi sur les investissements universitaires. Face aux difficultés immobilières de l'UQAM, le gouvernement exigea que les universités déclarent tout projet d'investissement et précisent la proportion de fonds publics et privés investis. Il y eut également d'autres tentatives avortées de modifier la gouvernance interne des universités et des cégeps, notamment en ce qui concerne la composition de leur conseil d'administration pour faire une plus grande place à des membres externes.

En 2012, l'élection du Parti Québécois mena à l'organisation d'un Sommet sur l'enseignement supérieur, suivi de cinq chantiers sur une loi-cadre sur les universités, la création d'un Conseil des universités, une nouvelle politique de financement, l'offre de formation au collégial et l'aide financière aux étudiants. Le gouvernement libéral qui lui a succédé a repris l'idée d'un Conseil des universités qui contribuerait à l'orientation générale du système et évaluerait les activités universitaires (Ministère de l'Éducation et de l'Enseignement supérieur, 2016).

En somme, pour Jamet (2014), le Québec possède un système d'enseignement supérieur centralisé et où le contrôle gouvernemental s'accroît au nom d'une plus grande imputabilité, mais dans lequel les universités ont plus d'autonomie que leurs homologues françaises. Comparant le Québec aux autres provinces canadiennes, Fisher et Rubenson (2014) y voient une continuité dans les intentions des gouvernements québécois qui se sont succédé de coordonner le système d'enseignement supérieur par diverses politiques, mesures budgétaires et organismes chargés d'évaluer les universités. 
En s'appuyant sur une analyse des modalités de gouvernance des systèmes d'enseignement universitaire, cet article vise à mettre en lumière diverses dimensions de l'encadrement du système universitaire québécois par l'État relativement à l'autonomie des établissements et à comparer la situation québécoise avec celle observée dans quatre autres provinces canadiennes.

\section{Cadre conceptuel}

Le concept de gouvernance renvoie à de multiples phénomènes, allant de l'instauration de partenariats public-privé (Rhodes, 1996) au remplacement de l'action unilatérale de l'État par un mode décisionnel consensuel et pluraliste (Pitseys, 2010). La gouvernance peut ainsi faire référence à une conception de l'action publique selon laquelle le pouvoir s'exerce de manière flexible dans un environnement où la société civile, les entreprises et l'État constituent des réseaux d'acteurs autonomes, mais interdépendants (Burlone et Rouillard, 2011). Dans le monde de l'enseignement supérieur, le concept de gouvernance renvoie " à la façon selon laquelle les systèmes d'enseignement supérieur et les établissements universitaires sont organisés et gérés, à la façon selon laquelle l'autorité y est distribuée et exercée, et à leurs relations avec les gouvernements » (Harman, 1991, p. 1280) [traduction des auteurs].

Il fautégalement reconnaître que la gouvernances'opèreà différents niveaux: lepremier niveau (micro) est celui des départements, le second (méso), celui des établissements, et le troisième (macro), celui du système d'enseignement supérieur dans son ensemble (Austin et Jones, 2016). La gouvernance systémique se distingue ainsi de la gouvernance interne des établissements en ce qu'elle a trait plus particulièrement à l'aménagement des relations entre les universités et les acteurs externes, notamment l'État, qui font partie de leur environnement. Il est d'autant plus pertinent d'analyser cette dimension de la gouvernance qu'elle se situe au cœur des processus décisionnels qui façonnent la configuration de l'enseignement universitaire.

Le concept de gouvernance systémique permet aussi de projeter un éclairage sur deux enjeux qui lui sont sous-jacents. Le premier a trait à l'autonomie institutionnelle, c'està-dire à la liberté des établissements de s'organiser pour remplir leurs missions de façon effective (Thorens, 2006). En 2007, l'Association des universités européennes (2007) a adopté la Déclaration de Lisbonne qui identifie quatre dimensions de l'autonomie institutionnelle : l'autonomie académique réfère à la capacité des universités de développer leur offre de formation et de recherche ; l'autonomie budgétaire renvoie à leur capacité de gérer les budgets qui leur sont alloués ; l'autonomie organisationnelle a trait aux procédures et structures internes qu'elles peuvent créer ou abolir ; et l'autonomie en matière de dotation se rapporte aux responsabilités qu'elles assument quant au recrutement, aux salaires et à l'avancement de leurs personnels. L'étendue de ces dimensions de l'autonomie est toutefois limitée par l'environnement externe des universités. Ces dernières essaient, en effet, de répondre aux attentes des acteurs externes afin d'obtenir le support et les ressources nécessaires à leur développement tout en sauvegardant leur autonomie institutionnelle et la liberté universitaire des professeurs (soit le droit et devoir des professeurs de développer des connaissances est de les diffuser sans contrainte). 
Le deuxième enjeu concerne l'imputabilité des établissements dans la mesure où les ressources dont ils disposent dépendent de l'État auquel ils sont redevables. La notion d'imputabilité renvoie plus spécifiquement à la responsabilisation des établissements dans l'exercice de leur mandat, à leur efficacité, à leur efficience et à la nécessité pour les établissements de rendre des comptes (Léveillé, 2013). Le concept de gouvernance implique également un certain degré de coordination, soit l'action de combiner des activités distinctes réalisées par différents acteurs en vue de constituer un ensemble cohérent (Bouchard, 2005).

À la lumière de ces concepts, notre objectif est de comparer les interactions entre les établissements universitaires et l'État dans le système d'enseignement universitaire québécois et de les comparer avec celles ayant cours dans quatre autres provinces en faisant le postulat que ces interactions influencent le degré d'autonomie institutionnelle. Afin de rendre notre comparaison plus systématique, nous identifierons certaines dimensions de la gouvernance systémique, soit des dispositions macroscopiques qui structurent les interactions entre l'État et les universités. Suivant une logique structuraliste, Kaplan (2004) fait le postulat que l'étude des structures de processus décisionnels permet de prédire les comportements organisationnels des acteurs.

Il n'existe cependant pas, à ce jour, de catégorisation de ces dimensions qui soit indépendante du contexte des pays étudiés. Par exemple, en se basant sur les cas de l'Allemagne, de l'Angleterre, de la France et de la Norvège, Bleiklie et Kogan (2007) ont identifié cinq structures nationales de gouvernance, soit les lois, les règlements et les politiques ; les formules de financement; les canaux de communication entre les universités et l'État; et les systèmes d'évaluation. Agasisti et Catalano (2006) ont catégorisé différents modèles de systèmes de gouvernance en étudiant les cas de l'Allemagne, de l'Espagne, de la France, de l'Italie et du Royaume-Uni. Les dimensions qu'ils ont retenues étaient la diversité institutionnelle, les processus d'accréditation des cours et des établissements, l'aide financière aux étudiants, la capacité des étudiants de choisir leur établissement et les mécanismes de financement des universités. Au Canada, Jones (2002) a, lui aussi, adopté une approche structuraliste des réseaux politiques dans le but de décrire les structures formelles de gouvernance et leur interprétation par les acteurs universitaires. Bien que l'étude portât d'abord sur la gouvernance interne des établissements, Jones s'est attardé à deux dimensions systémiques, soit le cadre législatif et les réseaux politiques (ex. : conseils d'administration, administrateurs, syndicats et associations étudiantes).

Tel qu'il sera mentionné ci-après, ces multiples catégorisations ont guidé l'élaboration de notre schéma d'entrevue et de notre grille d'analyse documentaire. Suivant une approche inductive, c'est toutefois le contenu des entretiens et des documents officiels analysés qui nous a permis de définir les dimensions de la gouvernance systémique des universités au Québec et au Canada. Ce faisant, l'article vise à répondre aux questions suivantes : (1) comment les dimensions sous-jacentes à la gouvernance de l'enseignement universitaire au Québec structurent-elles les interactions entre l'État et les universités ? et (2) comment le niveau d'autonomie relative des universités québécoises découlant de leurs relations avec l'État se compare-t-il à celui observé dans quatre autres provinces canadiennes? 


\section{Méthodologie}

Cet article émane d'un projet de recherche plus vaste sur la gouvernance qui s'appuie sur les études de cas de six grandes universités dans cinq provinces (Université de l'Alberta, Université de Colombie-Britannique, Université Dalhousie, Université de Montréal, Université du Québec à Montréal et Université de Toronto). Deux universités québécoises ont été sélectionnées afin de tenir compte des cadres législatifs différents qui régissent les universités à charte et le réseau de l'Université du Québec.

Le corpus de données fut colligé entre 2012 et 2014 et, tel que le montre le tableau 1, est composé de documents officiels et d'entrevues semi-structurées. Au total, nous avons sélectionné 42 documents provinciaux (ex. : lois, règlements, politiques et rapports) et 35 documents institutionnels (ex. : règlements, procédures, histoire de l'établissement et rapports), dont 29 concernant le Québec. Ces documents officiels étaient publiquement accessibles, devaient être effectifs au moment de l'analyse et faisaient référence au statut des universités, à leurs droits et obligations, à leur gestion interne et/ou à leurs relations avec d'autres organisations dans la province.

Tableau 1. Participants interviewés et documents analysés

\begin{tabular}{lcccrrr}
\hline Données/Provinces & Alberta & $\begin{array}{l}\text { Colombie- } \\
\text { Britannique }\end{array}$ & $\begin{array}{l}\text { Nouvelle- } \\
\text { Écosse }\end{array}$ & Ontario & Québec & Total \\
\hline Documents officiels & & & & & & \\
$\quad$ Documents institutionnels & 3 & 6 & 7 & 7 & 12 & 35 \\
$\quad$ Documents provinciaux & 6 & 8 & 5 & 6 & 17 & 42 \\
$\quad$ Total & 9 & 14 & 12 & 13 & 29 & 77 \\
Participants & & & & & & \\
$\quad$ Niveau institutionnel & 11 & 9 & 14 & 12 & 22 & 68 \\
$\quad$ Niveau provincial & 1 & 1 & 4 & 4 & 8 & 18 \\
$\quad$ Sous-total & 12 & 10 & 18 & 16 & 30 & 86 \\
$\quad$ Niveau fédéral & & & & & & 7 \\
$\quad$ Grand total & & & & & 93 \\
\hline
\end{tabular}

Au total, 93 entrevues (dont 30 au Québec) ont été menées avec des acteurs œuvrant à un niveau institutionnel, provincial ou fédéral. Le niveau fédéral comprend des représentants du gouvernement, des organismes subventionnaires et d'une association d'université. Le niveau provincial comprend des représentants des gouvernements, des organismes publics et parapublics, des représentants d'associations de professeurs et d'associations étudiantes. Le niveau institutionnel comprend des hauts-officiers d'universités, des membres des conseils d'administration, des représentants des professeurs et des étudiants, de même que des professeurs siégeant sur des instances décisionnelles. Une liste comprenant l'ensemble des acteurs nommés ci-haut fut élaborée pour chacune des provinces et, suivant les approbations éthiques et administratives, les participants furent invités, par courriel, à participer à une entrevue d'une heure. Les entrevues portaient sur le parcours et les fonctions de l'interviewé, les changements observés dans la gouvernance universitaire, les particularités des universités par rapport à d'autres types d'organisations, 
les rapports entre, d'une part, l'autonomie institutionnelle et la liberté universitaire et, d'autre part, les exigences des gouvernements et les mesures de reddition de comptes.

L'analyse documentaire - qui consistait à identifier les éléments relatifs aux interactions entre l'État et les universités - a débuté avant les entrevues afin de peaufiner les questions, puis s'est poursuivie afin d'appuyer les propos des participants. Les documents et les transcriptions furent analysés grâce à une analyse thématique (Braun et Clarke, 2006 ; Chaumier, 1997 ; Paillé et Mucchielli, 2012) qui permet d'identifier et d'analyser les schémas de réponse dans un corpus de données. Des thèmes de base furent identifiés dans les documents et dans les transcriptions d'entrevues, puis regroupés en dix thèmes centraux (ou indicateurs) reliant les principales idées aux questions de recherche. Finalement, ces dix thèmes furent regroupés en trois thèmes généraux qui devinrent, au terme de notre analyse, les dimensions de la gouvernance systémique.

\section{Dimensions de la gouvernance du système d'enseignement supérieur québécois}

L'analyse concomitante des documents et des entrevues a permis de dégager dix indicateurs relatifs à la gouvernance, qui témoignent des relations entre les universités et l'État, du contrôle que ce dernier exerce et de son impact sur l'autonomie des établissements. Ces indicateurs, formulés sous forme de questions, sont :

1. Existe-t-il une loi unique pour l'ensemble des universités ?

2. Le gouvernement considère-t-il que les universités font partie du secteur public ?

3. Les universités font-elles partie du périmètre comptable du gouvernement ?

4. Le vérificateur général agit-il comme auditeur externe des universités ?

5. Les règles budgétaires relatives aux subventions de l'État influencent-elles les décisions prises par les hauts administrateurs des universités?

6. Le gouvernement nomme-t-il les recteurs des universités ?

7. Le gouvernement nomme-t-il les membres des conseils d'administration (CA) des universités?

8. Le gouvernement interagit-il directement avec les membres du CA ?

9. Le gouvernement approuve-t-il les nouveaux programmes universitaires ?

10. Le gouvernement évalue-t-il les programmes existants?

Comme il a déjà été indiqué, c'est à partir du regroupement de ces thèmes qu'ont été construites trois grandes dimensions de la gouvernance systémique. La première a trait au cadre législatif et réglementaire, soit à la façon selon laquelle les interactions entre l'État et les universités sont formellement structurées par des chartes et des lois. Cette dimension rappelle l'une des cinq structures nationales de gouvernance de Bleiklie et Kogan (2007) et la dimension " cadre législatif » identifiée par Jones (2002). La seconde dimension concerne le statut comptable et les règles budgétaires définies par l'État. Elle renvoie à l'intégration ou non des universités dans le périmètre comptable du gouvernement, à la vérification de la comptabilité des établissements par le vérificateur général et au cadre budgétaire qui oriente les activités universitaires. Les formules et mécanismes de financement ont aussi été identifiés par Bleiklie et Kogan (2007), de même que par Agasisti et Catalano (2006). La troisième dimension porte sur les interventions du gouvernement dans la gestion institutionnelle des établissements, tel que la nomination des administrateurs, l'élaboration et l'évaluation des programmes. Cette 
dimension partage certaines similitudes avec les concepts d'autonomie organisationnelle et d'autonomie académique, tels que définis par l'Association européenne des universités (2007), de même qu'avec la dimension " accréditation des programmes » dans l'étude d'Agasisti et Catalano (2006).

\section{Cadre législatif et réglementaire}

Le système d'enseignement supérieur québécois se distingue des autres provinces par le fait que les universités opèrent selon deux cadres législatifs distincts : les huit universités les plus anciennes sont régies par leur propre charte (ou loi privée) alors que neuf constituantes de l'Université du Québec (UQ) et une université qui lui est associée (UQÀM) le sont par la loi quil'a instituée. En plus d'établir les fondements de la gouvernance interne des établissements, ces dispositions législatives circonscrivent le degré de leur autonomie par rapport au gouvernement. Par exemple, la Charte de l'Université de Montréal (1967) l'autorise à décerner des diplômes, à s'affilier par contrat à toute institution, à acquérir des immeubles et à nommer ses officiers. La Loi sur l'Université du Québec (2017) garantit à ses constituantes la liberté universitaire, leur permet de disposer de leurs biens, mais elles doivent obtenir l'autorisation du ministre pour la création d'écoles supérieures, sont gouvernées par une assemblée des gouverneurs dont les membres sont nommés par le gouvernement et doivent assurer la formation des enseignants. De plus, les chartes privées sont plus rarement revues par les parlements provinciaux, sauf à la demande explicite des établissements, comme dans le cas de l'Université de Montréal (UdeMNouvelles, 2017).

Par ailleurs, des hauts-officiers d'universités et des représentants du gouvernement ont confirmé, en entrevue, que les constituantes de l'UQ possédaient une autonomie légale plus restreinte que les universités à charte, mais aussi que l'UQÀM jouissait d'une plus grande autonomie que les autres composantes, puisqu'à titre d'université associée, elle pouvait décerner ses propres diplômes, recommander directement au ministre la nomination de son recteur et désigner ses hauts-officiers.

Qu'elles soient régies par des chartes privées ou par la Loi sur l'Université du Québec, l'autonomie des universités est contrainte par 52 lois québécoises (Bureau de coopération interuniversitaire, 2013), dont la Loi sur les investissements universitaires et la Loi sur les établissements de niveau universitaire. Cette dernière a d'ailleurs instauré une dimension de la gouvernance particulière au système québécois, soit l'obligation pour les universités, à l'instar des organismes publics, de rendre des comptes notamment au gouvernement et à la Commission de la culture et de l'éducation de l'Assemblée nationale et, pour les recteurs, de répondre en personne aux questions des membres de la Commission.

Il ressort aussi de l'analyse documentaire que l'État québécois associe les universités au secteur public. La Politique québécoise à l'égard des universités indique qu'elles constituent un « service public » qui « contribue à la réalisation de la mission gouvernementale d'éducation » (Gouvernement du Québec, 2000, p. 17). Par ailleurs, la Loi sur l'Assemblée nationale stipule qu'un député ne reçoit pas d'indemnité de départ s'il quitte son siège pour travailler dans une université (LRQ, chapitre A.23-1, aa.104, 124.2) et la Loi sur la Régie du logement inclut dans le secteur public tout établissement d'enseignement de niveau universitaire (Annexe III, chapitre E-14.1). En accord avec une conception du caractère " public » des universités, un haut-fonctionnaire interviewé a 
d'ailleurs expliqué s'attendre à ce que les universités utilisent judicieusement les fonds publics et contribuent au développement et à l'essor économique de la société québécoise.

Or, si tous les interviewés reconnaissaient qu'en vertu du contrat social établi lors de la Révolution tranquille (Vidricaire, 1996), les universités s'acquittent de responsabilités fondamentales pour la société en échange d'un financement public, les acteurs institutionnels et étatiques ne s'entendaient pas sur leur appartenance au secteur public. Une haute-officière universitaire a dénoncé ce qu'elle percevait comme un glissement :

Le ministre de l'Enseignement supérieur a confirmé qu'il concevait les universités comme faisant partie du secteur public. Une société d'État appartient au gouvernement, mais une université n'est pas sous l'autorité du ministre qui lui dit quoi faire et quoi penser ! C'est une institution publique, mais ce n'est pas une institution du secteur public.

Selon Levy (1986), le caractère public d'une université dépend de facteurs tels que le statut légal, le contrôle de l'université par des agents de l'État, les modalités de financement et l'usage courant. Au Québec, les universités sont largement soutenues par les deniers publics et, selon l'usage courant, appartiennent au " service public ». Quatre hautsofficiers et administrateurs d'universités se sont toutefois référé au cadre législatif (c.-à-d. les chartes privées) et au statut comptable (c.-à-d. l'exclusion du périmètre comptable du gouvernement) afin d'affirmer le caractère distinct de certaines universités par rapport à d'autres institutions, telles que les commissions scolaires, cégeps et hôpitaux. Ils suggéraient, par conséquent, que les constituantes du réseau de l'Université du Québec étaient plus assimilables au secteur public que ne le sont les universités à charte.

\section{Statut comptable et règles budgétaires relatives aux subventions de l'État}

Selon le Conseil sur la comptabilité dans le secteur public (2017) de l'Institut Canadien des Comptables Agréés, le périmètre comptable du gouvernement (PCG) englobe les organisations qu'il contrôle. Ce dernier repose sur sa capacité de nommer les membres du conseil d'administration d'une organisation, d'accéder à ses actifs, d'infléchir l'utilisation de ses ressources et d'assumer la responsabilité de ses pertes financières. Au Québec, les universités à charte sont exclues du PCG alors que les constituantes de l'UQ y sont incluses. Pour le Secrétariat du Conseil du trésor (2017), cette inclusion confirme l'appartenance de l'UQ au secteur public et limite son autonomie financière. Rappelons toutefois que toutes les universités sont tenues de faire approuver les projets d'investissement d'une valeur de plus d'un million de dollars (LRQ, c.I-17). Cette dernière disposition fut adoptée à la suite des difficultés immobilières relatives à un projet immobilier de l'UQÀM. Ces difficultés furent d'ailleurs documentées par le Vérificateur général du Québec (2008) qui, à l'époque, avait agi en vertu d'un décret gouvernemental. Depuis les modifications de 2008 à la Loi sur le vérificateur général, ce dernier peut agir à sa discrétion à titre d'auditeur externe des états financiers de l'UQ et de ses constituantes qui sont incluses dans le PCG.

Toutes les universités doivent aussi se conformer à des règles budgétaires gouvernementales précises liées à une subvention générale (basée sur des indicateurs relatifs àl'effectifétudiant, au soutien àl'enseignement età la recherche, et aux bâtiments) et 
à des subventions spécifiques (ex. : services aux étudiants, aux membres des communautés autochtones ou aux personnes handicapées). De 1999 à 2003, une subvention spécifique était liée à un contrat de performance entre chacun des établissements et le gouvernement. Ceux-ci ont été remplacés par une subvention conditionnelle à l'engagement des établissements à ne pas faire de déficit et à l'atteinte d'objectifs fixés par le gouvernement. Une analyse des politiques de financement et des cadres budgétaires nous amène à poser l'hypothèse que ces subventions ne contraindraient pas moins l'autonomie institutionnelle des établissements que les contrats de performance ne le faisaient puisque, contrairement à ces derniers, elles sont plus nombreuses, plus précises, moins adaptées au contexte de chacun et exigent une reddition de comptes dans un horizon temporel plus court. Finalement, le gouvernement fixe le niveau des droits de scolarité et encadre les frais institutionnels obligatoires. Si les universités dérogent à ce cadre, les montants perçus sont retranchés de la subvention générale (Ministère de l'Éducation et de l'Enseignement supérieur, 2017).

\section{Interventions du gouvernement dans la gestion institutionnelle des universités}

Cette dernière dimension regroupe quatre indicateurs qui relèvent de la gestion exécutive ou académique de l'université, soit la nomination du recteur, la nomination des membres des conseils d'administration (CA), les interactions entre l'État et le CA et l'approbation et l'évaluation des programmes universitaires. Le gouvernement ne nomme pas le recteur de l'Université de Montréal, mais désigne celui de l'UQÀM sur la recommandation du CA à la suite d'une consultation des personnes et des groupes institutionnellement reconnus. Pour un haut-officier, cette intervention du gouvernement n'affecte donc pas l'autonomie du recteur puisqu'il est d'abord élu par la communauté universitaire et a pour responsabilité, face à la population et au gouvernement, de défendre l'autonomie de l'université et la liberté universitaire. À noter qu'au Québec, les recteurs sont les principaux interlocuteurs auprès de l'État. Le gouvernement nomme néanmoins le tiers des membres du CA de l'Université de Montréal et tous ceux de l'UQÀM, suivant un processus de consultation ou d'élection (Université de Montréal, 2017 ; Université du Québec à Montréal, 2018).

En ce qui a trait à l'approbation et à l'évaluation des programmes, la CREPUQ assumait les responsabilités d'évaluer les projets de programme et de superviser l'évaluation des programmes existants. Succédant à la CREPUQ en 2013, le Bureau de coopération interuniversitaire (Bureau de coopération interuniversitaire, 2017) a repris l'évaluation des projets de programmes, mais pas la vérification des mécanismes d'évaluation des programmes existants par les établissements ; au moment d'écrire ces lignes, cette dernière responsabilité n'est assumée par aucun organisme, mais pourrait être dévolue à un éventuel Conseil des universités. Le Ministère de l'Éducation et de l'Enseignement supérieur (MEES) dispose aussi d'un comité des programmes universitaires dont le mandat est d'évaluer l'opportunité socio-économique, socioculturelle, systémique et institutionnelle de financer l'effectif étudiant des programmes. 


\section{Comparaison du système de gouvernance des universités québécoises avec ceux d'autres provinces canadiennes}

L'analyse a d'abord consisté à décrire les dimensions qui structurent les interactions entre l'État et les universités au Québec en vue de cerner les relations entre le contrôle exercé par l'État et l'autonomie des établissements. L'objectif de cette section est de se référer aux mêmes dimensions afin de comparer la situation québécoise à celles de l'Alberta, de la Colombie-Britannique, de l'Ontario et de la Nouvelle-Écosse. Bien que le Tableau 2 présente les résultats relatifs à tous les indicateurs utilisés dans le cas du Québec, cette section se limite à décrire les différences les plus significatives entre les provinces. L'article d'Eastman, Jones, Trottier, Bégin-Caouette, Li et Noumi (sous presse) présente une comparaison plus détaillée.

Le premier point de comparaison a trait au cadre législatif. En Nouvelle-Écosse et en Ontario, les universités opèrent selon des chartes privées. En Alberta et en Colombie-Britannique, elles le font, dans le premier cas, en fonction d'une loi unique sur l'enseignement postsecondaire et, dans le deuxième, d'une loi unique sur le système universitaire. Au Québec, l'UQ et ses constituantes sont régies par une loi unique alors que les autres disposent de leur charte privée. Comme au Québec, les entrevues réalisées dans les autres provinces révèlent que les universités opérant sous une loi unique ont moins d'influence sur le moment ou la direction des changements législatifs concernant leur gouvernance.

Selon des acteurs tant universitaires que gouvernementaux interviewés, les universités doivent composer, dans leur province respective, avec un accroissement des mécanismes d'imputabilité. Plusieurs représentants des gouvernements expliquaient cette situation par la nécessité pour les universités d'être sensibles aux priorités politiques du gouvernement et aux exigences de reddition de comptes. En Ontario, un acteur universitaire a indiqué que, « la province devient plus interventionniste ; ils ne nous donnent pas plus d'argent, mais nous règlementent davantage ». Un représentant du gouvernement notait également que, considérant les sommes investies par l'État, il y avait en Ontario, comme dans d'autres provinces, un nécessaire alignement des actions des universités sur les priorités du gouvernement. Selon un homologue albertain, il était justifié d'exiger des établissements postsecondaires qu'ils rendent des comptes aux contribuables considérant que $10 \%$ du budget opérationnel de la province leur était consacré. Les universités québécoises sont néanmoins les seules à devoir, selon la loi, rendre des comptes directement à leur assemblée législative provinciale.

Si des fonctionnaires de la Nouvelle-Écosse ont exprimé le plus clairement le souhait que les universités soient sensibles à la situation financière difficile de la province, ils reconnaissaient néanmoins que, dans de nombreux dossiers, elles ne faisaient pas partie du secteur public. À l'inverse, leurs homologues de l'Alberta, de la Colombie-Britannique, de l'Ontario et du Québec soulignaient diverses dispositions législatives qui témoignaient de leur appartenance au secteur public, par exemple l'obligation pour l'Université de Colombie-Britannique de faire approuver les indemnités versées au recteur ; l'assujettissement des universités britanno-colombiennes et québécoises à la divulgation salariale des dirigeants ; ou la nomination, par le gouvernement albertain, des présidents de conseil d'administration des universités. 


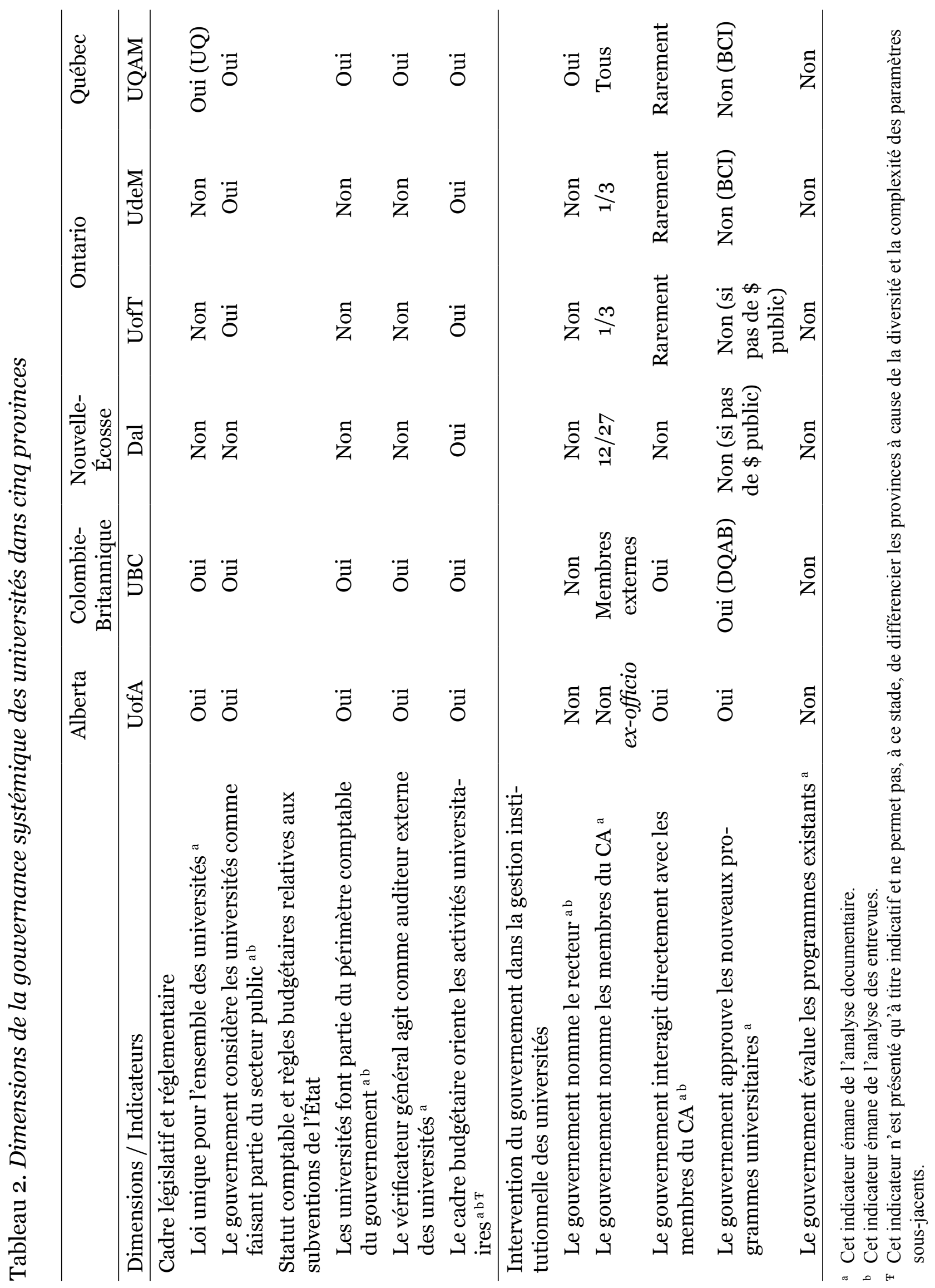


Le deuxième point de comparaison porte sur l'inclusion des universités dans le PCG. Historiquement, la plupart des universités canadiennes ne faisaient pas partie de ce périmètre. C'est encore le cas en Ontario, en Nouvelle-Écosse et au Québec (pour les universités à charte), mais ce ne l'est plus en Alberta et en Colombie-Britannique depuis 2003. De même, les vérificateurs généraux de l'Alberta, de la Colombie-Britannique et du Québec (dans le cas de l'UQ) agissent comme vérificateurs externes des universités. En Ontario, la Loi sur le vérificateur général de 2004 a élargi le mandat de vérification aux organismes du secteur parapublic, qui inclut les universités, mais ces dernières conservent le droit de faire examiner leurs états financiers par le vérificateur externe de leur choix.

La troisième différence concerne l'intervention étatique dans la gouvernance interne des établissements. Parmi les six universités qui ont fait l'objet des études de cas, seul le recteur de l'UQÀM est nommé par le gouvernement, bien que plusieurs autres universités admettent tenir le gouvernement informé du processus de nomination. Toutes les provinces nomment certains membres des CA universitaires, mais se distinguent quant à la proportion de membres nommés (ex. : le tiers à l'Université de Toronto et tous les membres de l'Université de l'Alberta qui ne sont pas membres d'office). Dans toutes les provinces, les universités soumettent une liste des candidats aux gouvernements. Cependant, alors que la Charte de l'Université Dalhousie oblige le gouvernement à choisir un candidat parmi cette liste, un officier universitaire ontarien a indiqué qu'il arrivait que le gouvernement de sa province déroge à cette pratique.

Cette différence est liée au type d'interaction entre les gouvernements et les CA. Dans les universités de l'Alberta et de la Colombie-Britannique - où le gouvernement intervient plus activement dans la nomination des membres -, le gouvernement cherche davantage à établir des canaux de communication formels avec les présidents des CA. En 2008, par exemple, le comité Campus Alberta était composé du ministre et des présidents de CA des universités et autres établissements postsecondaires. De même, en Alberta, comme en Colombie-Britannique, le gouvernement a exigé que ce soient les présidents des CA qui signent les lettres définissant les priorités des universités. Bien que les gouvernements de Nouvelle-Écosse, de l'Ontario et du Québec aient tenté d'établir de tels canaux de communication avec les CA, les recteurs demeurent, officiellement, les principaux interlocuteurs de leur établissement.

En ce qui a trait à l'approbation et l'évaluation des programmes, il y a lieu de distinguer les projets de programmes des programmes existants. Les universités albertaines doivent faire approuver leurs nouveaux programmes par le gouvernement, alors que celles de Colombie-Britannique devaient, au moment des entrevues, soumettre leur demande au Degree Quality Assessment Board et/ou au ministre, à moins d'exemptions. En Nouvelle-Écosse et en Ontario, les universités pouvaient créer de nouveaux programmes à condition qu'ils ne requièrent pas de financement public. Au Québec, la CREPUQ assumait la responsabilité d'évaluer les projets de programme jusqu'au moment de sa dissolution en 2013. Le Bureau de coopération interuniversitaire (Bureau de coopération interuniversitaire, 2017) qui lui a succédé a confié cette responsabilité à son comité d'évaluation des projets de programmes. Le MEES dispose aussi d'un comité qui évalue la pertinence de financer l'effectif étudiant des programmes universitaires. Pour ce qui est des programmes existants, l'Alberta, la Colombie-Britannique, la NouvelleÉcosse et l'Ontario s'assuraient, au moment des entrevues, que les universités avaient 
des mécanismes d'évaluation de ces programmes. Au Québec, cette responsabilité était auparavant dévolue à la Commission de vérification des programmes de la CREPUQ, dont le mandat était de vérifier si la mise en œuvre des politiques des établissements relatives à l'évaluation périodique des programmes était conforme à la politique générale à cet égard. Son mandat n'a pas été reconduit au sein du BCI.

Enfin, en marge des dimensions analysées, un quatrième point de comparaison a émergé au cours de l'analyse de certaines entrevues. Dans toutes les provinces, plusieurs participants ont dénoncé à la fois l'accroissement des mesures d'imputabilité et l'absence de rétroaction du gouvernement. Le Québec est néanmoins la seule province où des représentants des associations étudiantes, des syndicats de professeurs et même d'autres organismes provinciaux ont souhaité une plus grande coordination du système d'enseignement supérieur.

En somme, les données recueillies au moyen del'analyse documentaire et des entretiens (Tableau 2) permettent d'avoir une première approximation du degré d'autonomie des universités et de situer les provinces sur un continuum allant de l'autonomie institutionnelle au contrôle étatique. Une prochaine étude pourrait systématiser cette analyse comparative, mais, déjà, nous constatons que les systèmes de gouvernance universitaire de la Nouvelle-Écosse et de l'Ontario se situeraient plus près du pôle « autonomie », alors que ceux de la Colombie-Britannique et l'Alberta se trouveraient plus près du pôle " contrôle étatique ». Ce modèle permet de jeter un éclairage nouveau sur les particularités du système universitaire québécois qui, bien qu'étant unifié par plusieurs lois, règles budgétaires et organismes communs (le Conseil supérieur de l'éducation et le $\mathrm{BCI}$ ), demeure composé de deux types d'universités, dont les premières (universités à charte) se situeraient plus près du pôle « autonomie » et les secondes (constituantes de l'UQ) du pôle « contrôle étatique ». Par ailleurs, les universités à charte jouiraient d'une autonomie plus limitée que celle dont bénéficie, par exemple, l'Université Dalhousie parce que le gouvernement les considère, à l'instar de l'UQ, comme appartenant au secteur public, édicte des règles budgétaires et de gestion immobilière strictes et leur demande de rendre des comptes à l'Assemblée nationale. Les constituantes de l'UQ, de leur côté, jouiraient peut-être, et à certains égards, d'une plus grande autonomie que l'Université d'Alberta et l'Université de la Colombie-Britannique, étant donné qu'elles réclament un traitement équivalent à celui accordé aux universités à charte et que les deux types d'universités qui étaient jadis représentés par la CREPUQ le sont maintenant par le BCI. Ainsi, sans bénéficier du même cadre législatif, les constituantes de l'UQ profiteraient de certains avantages consentis aux universités à charte. Le gouvernement, par exemple, interagit directement avec les recteurs des UQ, comme il le ferait avec les recteurs des universités à charte, et non avec les présidents de $\mathrm{CA}$, comme le font les gouvernements de l'Alberta et de Colombie-Britannique. Les universités de ces deux provinces peuvent néanmoins conserver leurs surplus budgétaires, déterminer les salaires des hautsdirigeants et embaucher leur personnel de manière plus autonome que ne le peuvent les constituantes de l'UQ. Le Québec, dans son ensemble, se situerait donc au centre du continuum puisque, par souci d'équité ou sous la pression de la CREPUQ et du BCI, le gouvernement en est venu à traiter de la même façon l'ensemble des universités.

Finalement, nos résultats suggèrent que le niveau relatif d'autonomie universitaire varie d'une province à l'autre. Ces différences pourraient s'expliquer, en partie, par le 
développement historique de chacun des systèmes provinciaux. Harmsen et Tupper (2017) avaient en effet noté que l'évolution de ces derniers s'ancrait dans un contexte local cristallisé par des choix politiques antérieurs. Les universités néo-écossaises, ontariennes et les premières universités québécoises ont, pour la plupart, été fondées par des communautés religieuses, alors que la majorité de leurs homologues albertaines, britanno-colombiennes, de même que les constituantes de l'UQ, furent établies par l'État à titre de service public destiné à l'ensemble de la population. Bien qu'au Québec, tous les établissements doivent répondre aux mêmes exigences relatives à l'imputabilité, ceux de l'UQ, compte tenu du contexte historique de la Révolution tranquille dans lequel ils ont été créés, peuvent plus difficilement se soustraire au contrôle étatique.

\section{Conclusion}

L'objectif de cette étude était d'analyser la gouvernance systémique des universités québécoises en examinant leurs relations avec l'État québécois et d'en comparer les principales dimensions avec celles de la gouvernance systémique dans quatre autres provinces. L'analyse a montré que l'autonomie dont jouissent les universités varie d'une province à l'autre. Au Québec, les universités à charte privée bénéficient d'une plus grande autonomie formelle que les constituantes de l'UQ. La gouvernance de l'UQ a, en effet, plus de points en commun avec celle des universités de l'Alberta et de la ColombieBritannique, alors que celle des universités à charte québécoises ressemble davantage à celle des universités néo-écossaises et ontariennes.

Malgré ces différences, le système québécois n'en demeure pas moins unifié par plusieurs lois, règles budgétaires et organismes qui concernent les deux types d'universités. De plus, la dynamique qui est établie entre les universités à charte, l'UQ et le gouvernement témoigne du caractère distinct du système québécois. D'une part, les universités à charte jouissent d'une autonomie plus limitée que celle dont bénéficie l'Université Dalhousie parce que le gouvernement les considère toutes, à l'instar de l'UQ, comme appartenant au secteur public et, par conséquent, les soumet aux mêmes règles budgétaires ou relatives à leurs investissements et les oblige à rendre des comptes à l'Assemblée nationale. D'autre part, les constituantes de l'UQ parviennent à maintenir un certain degré d'autonomie en réclamant un traitement équivalent à celui accordé aux universités à charte et en étant représentées, à l'instar de celles-ci, par le BCI. Ces résultats suggèrent qu'il serait possible, dans une prochaine étude, de recourir à un cadre conceptuel plus élaboré qui, comme celui d'Esterman et al. (2011) pour l'Europe, permettrait de caractériser de façon plus exhaustive la gouvernance des systèmes d'enseignement universitaire.

Cetteétude n'est toutefois basée que sur six études de cas qui ne sont pas nécessairement représentatives de l'ensemble des universités canadiennes. Dû aux contraintes d'espace, il n'était pas, non plus, possible de présenter certaines nuances apportées par des représentants des associations étudiantes et des syndicats de professeurs quant aux distinctions entre imputabilité et coordination, ou entre liberté universitaire et autonomie institutionnelle.

De plus, au-delà du continuum allant du pôle " autonomie des établissements » au " contrôle étatique », une prochaine étude pourrait se pencher sur les impacts respectifs des mesures d'imputabilité et de coordination imposées aux établissements sur leur autonomie et la liberté universitaire. De même, un examen plus détaillé des modalités des 
règles budgétaires édictées par l'État permettrait d'avoir une idée plus précise du contrôle qu'il exerce et des mécanismes d'imputabilité auxquels il a recours. Enfin, il y aurait lieu d'examiner les relations entre les universités, les entreprises et les autres groupes de la société civile afin de comprendre comment elles parviennent à sauvegarder leur autonomie face aux attentes et demandes de ces derniers. Cet article constitue néanmoins une première étape dans la compréhension des interactions entre contrôle étatique et autonomie universitaire et des dimensions qui les structurent dans les contextes québécois et canadiens.

\section{Références}

Altbach, P.G. et Balán, J. (2007). World class worldwide: Transforming research universities in Asia and Latin America. Baltimore, MD: Johns Hopkins University Press.

Anderson, D. et Johnson, R. (1998) University autonomy in twenty countries (Research Report No. 98/3). Récupéré de http://www.magna-charta.org/resources/ files/University_autonomy_in_20_countries.pdf.

Agasisti, T. et Catalano, G. (2006). Governance models of university systems - towards quasi-markets? Tendencies and perspectives: An European comparison. Journal of Higher Education Policy and Management, 28(3), 245-262. doi: 10.1080/13600800600980056.

Association européenne des universités (2007). Déclaration de Lisbonne - Les universités européennes après 2010 : la diversité dans la poursuite d'un objectif commun. Bruxelles, Belgique : Association européenne des universités. Récupéré de http://www. eua.be/Libraries/quality-assurance/lisbon_declaration.pdf?sfvrsn=0.

Austin, I. et Jones, G. (2016). Governance of higher education: Global perspectives, theories, and practices. New York, NY: Routledge.

Bureau de coopération interuniversitaire (2017). Rapport annuel 2016-2017 déposé à l'Assemblée générale annuelle du 6 octobre 2017. Récupéré de http://www.bci-qc.ca/ wp-content/uploads/2017/11/Rapport-annuel-2016-2017.pdf.

Bureau de coopération interuniversitaire (2013). Liste des principales lois québécoises applicables aux établissements universitaires. Récupéré de https://www.bci-qc.ca/wpcontent/uploads/2016/10/Inventaire_lois_prov_applicables_univers_24juillet13.pdf.

Bleiklie, I. et Kogan, M. (2007). Organization and governance of universities. Higher Education Policy, 20(4), 477-493. https://doi.org/10.1057/palgrave.hep.8300167.

Braun, V. et Clarke, V. (2006). Using thematic analysis in psychology. Qualitative Research in Psychology, 3(2), 77-101.

Bouchard, M.J. (2005). Nouvelles formes de régulation et de coordination dans la gouvernance des entreprises collectives. Annales de l'économie publique et coopérative, 76(4), 581-584.

Burlone, N. et Rouillard, C. (2011). La reconfiguration de l'espace public par la gouvernance : innovation rhétorique ou changement paradigmatique ? Dans C. Rouillard et N. Burlone (dir.), L'État et la société civile sous le joug de la gouvernance (pp. 1-7). Québec, Canada : Presses de l'Université Laval. 
Chaumier, J. (1997). L'analyse documentaire ou la valorisation des documents. Recherche en soins infirmiers, 50(1), 99-102.

Conseil sur la comptabilité dans le secteur public (2017). 20 Questions sur le périmètre comptable du gouvernement. Toronto, Canada : Institut Canadien des Comptables Agréés. Récupéré de http://www.nifccanada.ca/normes-pour-les-entites-du-secteurpublic/ressources/documents-de-reference/item14972.pdf.

Commission des États généraux sur l'éducation (1996). Renouveler notre système d'éducation : Dix chantiers prioritaires. Québec : Gouvernement du Québec. Récupéré de http://collections.banq.qc.ca/ark:/52327/bs40260.

Conférence des recteurs et des principaux des universités du Québec (2013). Faire de l'enseignement supérieur une priorité nationale. Récupéré à http://www.newswire. $\mathrm{ca} /$ news-releases/faire-de-lenseignement-superieur-une-priorite-nationale-511976011. html.

Eastman, J., Jones, G.A., Trottier, C., Bégin-Caouette, O., Li, S. et Noumi, C. (sous presse). Provincial oversight and university autonomyin Canada: Findings of a comparative study of Canadian university governance. Canadian Journal of Higher Education/Revue canadienne d'enseignement supérieur.

Estermann, T., Nokkala, T. et Steinel, M. (2011). University Autonomy II: The Scorecard. Brussels, Belgium: European University Association. Récupéré de http:// www.eua.be/Libraries/publications/University_Autonomy_in_Europe_II_-_The_ Scorecard.pdf?sfvrsn=2.

Fisher, D. et Rubenson, K. (2014). Introduction and overview: PSE in three Canadian provinces. Dans D. Fisher, K. Rubenson, T. Shanahan, et C. Trottier (dir.), The Development of Postsecondary Education Systems in Canada: A Comparison Between British Columbia, Ontario, and Quebec 1980-2010 (pp. 3-34). Montreal \& Kingston: McGill-Queen's University Press.

Gouvernement du Québec (2000). Politique québécoise à l'égard des universités. Pour mieux assumer notre avenir collectif. Québec, Canada : Ministère de l'Éducation du Québec. Récupéré de http://www.education.gouv.qc.ca/fileadmin/site_web/ documents/enseignement-superieur/universitaire/Politique_quebecoise_universites_ Avenir_collectif_VF.pdf.

Gouvernement du Québec (1979a). Commission d'étude sur les universités - Comité d'étude sur l'organisation du systèmeuniversitaire, le réseau universitaire, l'organisation et la gestion de l'université et les étudiants à l'université (Rapport mai 1979). Québec, Canada : Gouvernement du Québec.

Gouvernement du Québec (1979b). Comité d'étude sur l'université et la société québécoise (Rapport mai 1979). Québec, Canada : Gouvernement du Québec.

Harman, G. (1991). Governance, administration and finance. Dans B.R. Clark et G.R. Neave (dir.), The encyclopedia of higher education, vol. 2. (pp. 1279-1293). Oxford, UK: Pergamon Press. 
Harmsen, R. et Tupper, A. (2017). The governance of post-secondary education systems in British Columbia and Ontario: Path dependence and provincial policy. Canadian Public Administration, 6o(3), pp. 349-368.

Jamet, M.(2014).Legouvernement des universités au Québec eten France:Conceptions de l'autonomie et mouvements vers un pilotage stratégique. Dans T. Chevaillier et C. Musselin (dir.), Réformes d'hier et réformes d'aujourd'hui : L'enseignement supérieur recomposé (pp. 21-59). Rennes, France : Presses universitaires de Rennes.

Jones, G.A. (2002). The structure of university governance in Canada: A policy network approach. Dans A. Amaral, G.A. Jones et B. Karseth (dir.), Governing higher education: National perspectives on institutional governance (pp.213-234). Dordrecht, The Netherlands: Kluwer Academic Publishers.

Kaplan, G.E. (2004). Do governance structures matter? New directions for higher education, 127(1), 23-35. doi.org/10.1002/he.153.

Lacroix, A. et Maheu, L. (2015). Les grandes universités de recherche. Montréal, Canada : Presses de l'Université de Montréal.

Lang, D.W. (2016). Incentive funding meets incentive-based budgeting: Can they coexist? Canadian Journal of Higher Education/Revue canadienne d'enseignement supérieur, 46(4), 1-22.

Lessard, C. (2006). La " gouvernance » de l'éducation au Canada : tendances et significations. Éducation et sociétés, 18(2), 181-201.

Levy, D.C. (1986). 'Private' and 'public': Analysis and ambiguity in higher education. Dans D.C. Levy (dir.) Private education: Studies in choice and public policy. (pp. 170192). New York, NY: Oxford University Press.

Metcalfe, A.S., Fisher, D., Gingras, Y., Jones, G.A., Rubenson, K. et Snee, I. (2011). Canada: Perspectives on governance and management. Dans W. Locke, W. Cummings, et D. Fisher (dir.), Changing Governance and Management in Higher Education (pp. 151174). Dordrecht, The Netherlands: Springer.

Ministère de l'Éducation et de l'Enseignement supérieur (2017). Règles budgétaires et calcul des subventions de fonctionnement aux universités du Québec - Année universitaire 2017-2018. Québec, Canada : Gouvernement du Québec. Récupéré de http://www.education.gouv.qc.ca/universites/professeurs-et-personnel-duniversite/ regles-budgetaires-et-reddition-de-comptes/calculs-des-subventions.

Ministère de l'Éducation et de l'Enseignement supérieur (2016). Projet de création du conseil des universités du Québec et de la Commission mixte de l'enseignement supérieur. Québec : Gouvernement du Québec. Récupéré de http://www.education.gouv. qc.ca/fileadmin/contenu/documents_soutien/Ens_Sup/Commun/Consultations_ES/ Document_consultation_Universites.pdf.

Paillé, P. et Mucchielli, A. (2012). L'analyse qualitative en sciences humaines et sociales. Paris : Armand Colin.

Pitseys, J. (2010). Le concept de gouvernance. Revue interdisciplinaire d'études juridiques, 65(2). doi: 10.3917/riej.065.0207. 
Richardson, G. et Fielden, J. (1997). Measuring the grip of the state: The relationship between governments and universities in selected Commonwealth countries. London, UK: Commonwealth Higher Education Management Service.

Rhodes, R.A.W. (1996). The new governance: Governing without government. Political Studies, XLIV, 652-667.

Secrétariat du Conseil du trésor (2017). Les effectifs des ministères, des organismes, des réseaux du secteur public et des sociétés d'État. Récupéré de https://www.tresor. gouv.qc.ca/fileadmin/PDF/effectif_gestion_controle/1501_1512.pdf.

Shanahan, T. et Jones, G.A. (2007). Shifting roles and approaches: Government coordination of post-secondary education in Canada, 1995-2006. Higher Education Research \& Development, 26(1), 31-43.

Shore, C. et Taitz, M. (2012). Who 'owns' the university? Institutional autonomy and academic freedom in an age of knowledge capitalism. Globalisation, Societies and Education, 10(2), 201-219.

Thorens, J. (2006). Liberties, freedom and autonomy: a few reflections on academia's estate. Higher Education Policy, 19(1), 87-110.

Trottier, C., Bernatchez, J., Fisher, D. et Rubsenson, K. (2014). PSE policy in Quebec: A case study. Dans D. Fisher, K. Rubenson, T. Shanahan et C. Trottier (Eds), The Development of Postsecondary Education Systems in Canada: A Comparison Between British Columbia, Ontario, and Quebec 1980-2010 (pp. 200-290). Montreal \& Kingston: McGill-Queen's University Press.

UdeMNouvelles (2017, 20 septembre). Charte de l'UdeM : le projet de loi est déposé à Québec. UdeMNouvelles. Récupéré de http://nouvelles.umontreal.ca/ article/2017/09/20/charte-de-l-udem-le-projet-de-loi-est-depose-a-quebec.

Université de Montréal (2017). Charte de l'Université de Montréal, 1967, 15-16 Eliz. II, chap. 129. Récupéré de http://secretariatgeneral.umontreal.ca/fileadmin/secretariat/ Documents/Documents_officiels/charte.pdf.

Vérificateur général du Québec (2008). Rapport du Vérificateur général du Québec à l'Assemblée nationale pour l'année 2008-2009 (Tome 1). Québec, Canada : Vérificateur général du Québec. Récupéré de http://www.vgq.gouv.qc.ca/fr/fr_publications/fr_ rapport-annuel/fr_2008-2009-T1/fr_Rapport2008-2009-T1-AnnexeA.pdf.

Vidricaire, A. (1996). Le syndicalisme universitaire dans son rapport avec le mouvement syndical. Dans G. Leroux et A. Vidricaire (dir.) Vingt-cinq ans de syndicalisme universitaire : Éléments d'histoire et enjeux actuels. Analyses et discussions (pp. 185192). Montréal, Canada : SPUQ.

Université du Québec à Montréal (2018). Règlement des procédures de désignation - Règlement numéro 3. Récupéré à https://instances.uqam.ca/wp-content/uploads/ sites/47/2017/12/REGLEMENT_NO_3-1.pdf. 


\section{Notices biographiques}

Olivier Bégin-Caouette

Département administration et fondements de l'éducation, Faculté des sciences de l'éducation, Université de Montréal

olivier.begin-caouette@umontreal.ca

Olivier Bégin-Caouette est professeur adjoint en enseignement supérieur comparé au Département d'administration et fondements de l'éducation de l'Université de Montréal. Il est membre associé du Centre interuniversitaire de recherche sur la science et la technologie (CIRST). Il œuvre également comme consultant auprès de l'Université de l'Ontario français (UOF). Ses intérêts de recherche portent sur les systèmes d'enseignement supérieur, la production de recherche universitaire et l'internationalisation. Pour plus d'informations : http://olivierbegincaouette.yolasite.com

Claude Trottier (Ph.D., Université de Toronto) est professeur émérite de la Faculté des sciences de l'éducation de l'Université Laval. Ses domaines de spécialisation sont la sociologie de l'éducation et l'analyse des politiques éducatives. Ses recherches portent sur les parcours scolaires des étudiants, la transition du système éducatif au marché du travail et l'analyse des politiques d'enseignement supérieur.

Julia Eastman, basée à Victoria, est chercheuse et praticienne dans le domaine de la gouvernance universitaire. De 2005 à juin 2018, elle a occupé le poste de secrétaire générale de l'Université de Victoria. Entre 1982 et 2003, elle a occupé divers postes administratifs à l'Université Dalhousie et, en 2004, est devenue directrice principale au ministère de l'Éducation de la Nouvelle-Écosse, division des collèges et des universités. Elle a auparavant travaillé au Conseil des premiers ministres des Maritimes et à l'Institut des relations internationales de l'Université Queen's. Julia détient un doctorat en enseignement supérieur de l'Université de Toronto. Avec Daniel Lang, elle a co-écrit un livre sur les fusions d'établissements d'enseignement supérieur.

Glen A. Jones est professeur titulaire en enseignement supérieur et doyen de l'Institut d'études pédagogiques de l'Ontario (OISE) de l'Université de Toronto. Ses recherches portent sur les systèmes d'enseignement supérieur, la gouvernance et le travail universitaire. Avec Ian Austin, il a co-écrit Governance of Higher Education: Global Perspectives, Theories and Practices (Routledge, 2015). Ses ouvrages plus récents comprennent Doctoral Education for the Knowledge Society (avec J.C. Chin et Barbara Kehm, Springer, 2018) et Professorial Pathways (avec Martin Finkelstein, John Hopkins University Press, 2019). Pour plus d'informations : www.glenjones.ca

Christian Noumi est candidat au doctorat en enseignement supérieur à l'Université de Toronto. Il possède un baccalauréat en histoire (Université de Yaoundé, Cameroun) et une maîtrise en recherche et développement de l'enseignement supérieur (Université de Cassel, Allemagne). Ses intérêts de recherche portent sur la comparaison de la gouvernance et des politiques d'enseignement supérieur. 
Sharon X. Li est animatrice de programmes au Continuing and Professional Learning de l'Institut d'études pédagogiques de l'Ontario (OISE), de l'Université de Toronto. Ses recherches portent sur l'enseignement supérieur comparé et la gouvernance universitaire. Elle détient un doctorat en enseignement supérieur de l'Université de Toronto. 